\title{
Support Epidemiology and Prognosis of Premature Rupture of Membranes in Pikine National Hospital Center
}

\author{
Moussa Diallo1, Abdoul Aziz Diouf', Hadja Maimouna Barro Daff²*, Natty Seck ${ }^{2}$, Aminata Niass ${ }^{1}$, \\ Youssou Toure ${ }^{2}$, Khalifa Fall ${ }^{2}$, Codou Sene Seck ${ }^{1}$, Alassane Diouf ${ }^{1}$ \\ ${ }^{1}$ Departement of Obstetrics and Gynecology Pikine University Hospital, Thiaroye Pikine, Sénégal \\ ${ }^{2}$ Cheikh Anta Diop University of Dakar, Gynecological and Obsetric Clinic, Public Institution Aristide-Le Dantec Health Center, \\ Dakar, Senegal \\ Email: ^hmbdaff@gmail.com
}

How to cite this paper: Diallo, M., Diouf, A.A., Daff, H.M.B., Seck, N., Niass, A., Toure, Y., Fall, K., Seck, C.S. and Diouf, A. (2019) Support Epidemiology and Prognosis of Premature Rupture of Membranes in Pikine National Hospital Center. Open Journal of Obstetrics and Gynecology, 9, 1519-1526.

https://doi.org/10.4236/ojog.2019.911147

Received: October 8, 2019

Accepted: November 16, 2019

Published: November 19, 2019

Copyright $\odot 2019$ by author(s) and Scientific Research Publishing Inc. This work is licensed under the Creative Commons Attribution International License (CC BY 4.0).

http://creativecommons.org/licenses/by/4.0/ (c) (i) Open Access

\begin{abstract}
Premature rupture of membranes (PROM) complicates $3 \%$ of preterm pregnancies and occurs in $60 \%$ to $80 \%$ of term pregnancies. However, its management remains largely controversial. The objective of this study was to establish the epidemiological profile, to study the management and the prognosis of Premature rupture of membranes (PROM) in our practice. Patients and methods: It was a prospective, descriptive and analytical study from May 1st 2016 to January 31st 2017 at the Pikine National Hospital Center. The target population consisted of all patients received at the hospital with premature rupture of membranes and who had given birth in the structure. The variables studied were: marital status, mode and reason for admission; risk factors; antecedents; prenatal care; the clinical and paraclinical examinations; support and immediate maternal and fetal neonatal complications. Results and comments: The mean maternal age was 27.34 years and the majority of women were aged between 18 and 39 years (94.4\%). Fifty-one point three percent of patients were primiparous, large multiparous represented only $2.5 \%$. The majority of patients (385 patients or $66.9 \%$ ) had consulted in the first 12 hours following the onset of fluid flow. For $20.1 \%$ of them this flow was associated with uterine contractions. Hidden risk factors were dominated by the twin pregnancy. The blood count showed that $38.8 \%$ of patients had leukocytosis and CRP was positive in $18.3 \%$ of patients. An ampicillin-based antibiotics was established in $42.6 \%$ of cases, corticosteroid therapy in $5.2 \%$ and $1 \%$ in tocolysis. An expectation was adopted in $65.7 \%$ of cases, induction of labor in $7.3 \%$ and a cesarean section immediately in $27 \%$ of cases. In total, $65.7 \%$ of patients had vaginal delivery and $34.3 \%$ cesarean. The perinatal mortality rate was $3.6 \%$ or 22 newborns on 610 . Two cases of endometritis
\end{abstract}


were observed and one case of immediate postpartum hemorrhage. No maternal deaths were recorded. Conclusion: These results show that the prognosis of premature rupture of membranes remains favorable in our practice. To improve this prognosis, we recommend sensitization of patients during prenatal care regarding signs of danger, a systematic bacteriological sample from all pregnant at the end of their pregnancy and the health personnel to direct patients' references to structures in case of PROM.

\section{Keywords}

Premature Rupture of Membranes, Childbirth, Stillbirth, Chorioamnionitis

\section{Introduction}

Premature rupture of membranes (PROM) is a relatively frequent pathology obstetric complicating $3 \%$ of preterm pregnancies [1] and occurring in $60 \%$ to $80 \%$ of term pregnancies [2]. It corresponds to an opening of the amniotic membranes before labor begins. It is the leading cause of premature delivery and neonatal death [3]. His etiology is multifactorial, although the lower genital tract infection is the factor most commonly found especially at an earlier gestational age. Other risk factors can be personal (age, parity, bad socioeconomic conditions, medical history), constitutional (uterine malformations: hypoplastic uterus) or traumatic. His diagnosis is easy and is based, in most cases, on an interview and a thorough clinical examination. But in some cases, the flow is intermittent and discreet and does not allow the diagnosis. Different biological examinations will then be carried out to make the diagnosis: vaginal fluid $\mathrm{pH}$, crystallization test, amnio dye test, fibronectin test, a 1-microglobulin protein (PAMG-1) study, growth factor research insulin or IGFBP1 [4] [5]. The management depends on several factors including gestational age, the time elapsed since the opening of the egg, the Bishop score, any associated pathologies and the technical platform available. Its aim is to improve maternal-fetal and neonatal prognosis minimizing complications of prematurity and infection.

The objectives of this study were to establish the epidemiological profile, to study the management and prognosis of this disease in our practice.

\section{Patients and Methods}

It was a prospective, descriptive and analytical study from May 1st 2016 to January 31 st 2017 at the Pikine National Hospital Center.

The target population consisted of all patients received at the hospital with premature rupture of membranes and who had given birth in the structure.

Data collection was done using individual questionnaires (survey form) from the hospital records, partographs, the register of births and the register of neonatology.

The variables studied were: marital status, mode and reason for admission; 
risk factors; antecedents; prenatal care; the clinical and paraclinical examinations; support and fetal maternal complications and immediate neonatal.

The data were received on individual survey forms. The data was analyzed with sphinx software and SPSS version 23 (IBM, United States) and descriptive statistics were used for frequency studies. The correlation tests were done by the Chi-2 test.

\section{Results}

\section{Frequency}

During the study 575 confirmed cases of PROM were reported on a total of 3003 women in labor, a frequency of $19.15 \%$.

\section{Socio-demographic characteristics \\ Age of patients}

The average age of patients was 27.34 years with extremes of 15 and 49. The majority of our patients (94.4\%) were aged between 18 and 39 years.

\section{Gravidity and parity}

Fifty-one-point three percent of patients were primiparous $(>6)$, large multiparous represented only $2.5 \%$.

\section{Level of education}

Almost half of our patients were not educated (49.7\%). The one who received a primary school education accounted for $27.6 \%$. Only $9.7 \%$ had completed higher education.

\section{Clinical aspects \\ Pregnancy}

The pregnancies were a singleton in the majority of the cases $(93.2 \%)$ while the twins were $6.25 \%$ of cases. The average gestational age was 38.7 weeks with extremes of 23 and 46 . The majority of patients (71.7\%) had a gestational age greater than 37 weeks.

\section{Number of antenatal}

The majority of patients had performed more than 3 prenatal consultations, $88 \%$ of cases. The average number of antenatal visits was 4 with a range of 0 to 9 . One-point six percent (1.6\%) of patients had not received prenatal care. However, there does not seem to be any relationship between the number of prenatal care and the occurrence of the PROM $(\mathrm{p}=0.577)$.

\section{Clinical data}

The referred patients accounted for $53.6 \%$ against $46.4 \%$ of spontaneous admissions. We note that the majority of patients (385 patients or $66.9 \%$ ) had consulted in the first 12 hours of the onset of fluid flow and only 94 patients (16.3\%) had consulted 24 hours later. The risk factors were dominated by the twin pregnancy (Table 1). The fluid flow was the most frequent reason for consultation (38.9\%). For $20.1 \%$ of them, this flow was associated with uterine contractions.

The majority of fetal presentations were cephalic with $86 \%$ (Table 2). The 
amniotic fluid was clear in $40.9 \%$ (Figure 1). In $88.9 \%$ of cases, clinical pelvimetry was normal. The shrunken basins (immature, limiting, generally and transversely narrowed) accounted for $8 \%$ of cases.

\section{Biology}

The C-reactive protein was performed in 160 patients or $27.8 \%$ and was higher in $18.3 \%$. The blood count was performed in $83.5 \%$ of patients, leukocytosis was noted in 223 patients or $38.8 \%$ of cases. Among the 223 patients with leukocytosis, 62 had elevated CRP. Anemia was present in 29 patients.

Among the 575 cases,

- 245 had received antibiotic therapy, a rate of $42.6 \%$;

- 30 patients received corticosteroids or $5.2 \%$ of cases;

- 6 patients were under tocolysis $1 \%$ of cases.

In our series, $65.7 \%$ of patients had received simple monitoring and $7.3 \%$ of patients had undergone induction of labor. Immediate caesarean section was performed in $27 \%$ of cases. The results are shown in Figure 2.

In total 197 patients had a cesarean section or $34.3 \%$, and 378 patients had vaginal delivery $65.7 \%$ of cases.

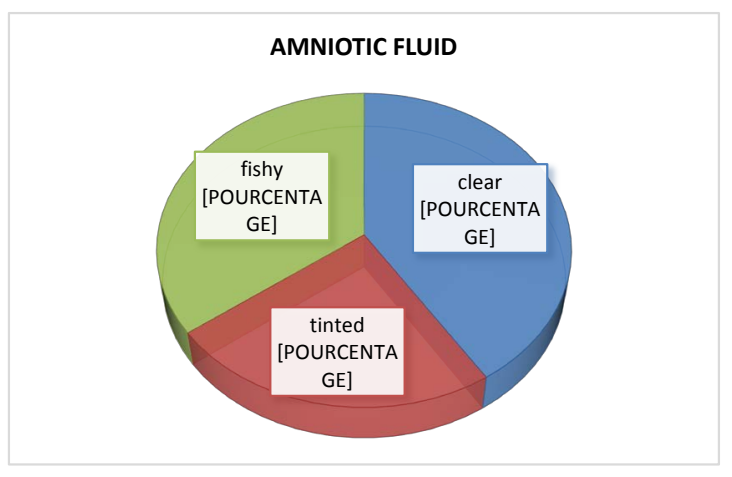

Figure 1. Distribution of patients according to the aspect of the amniotic fluid supported.

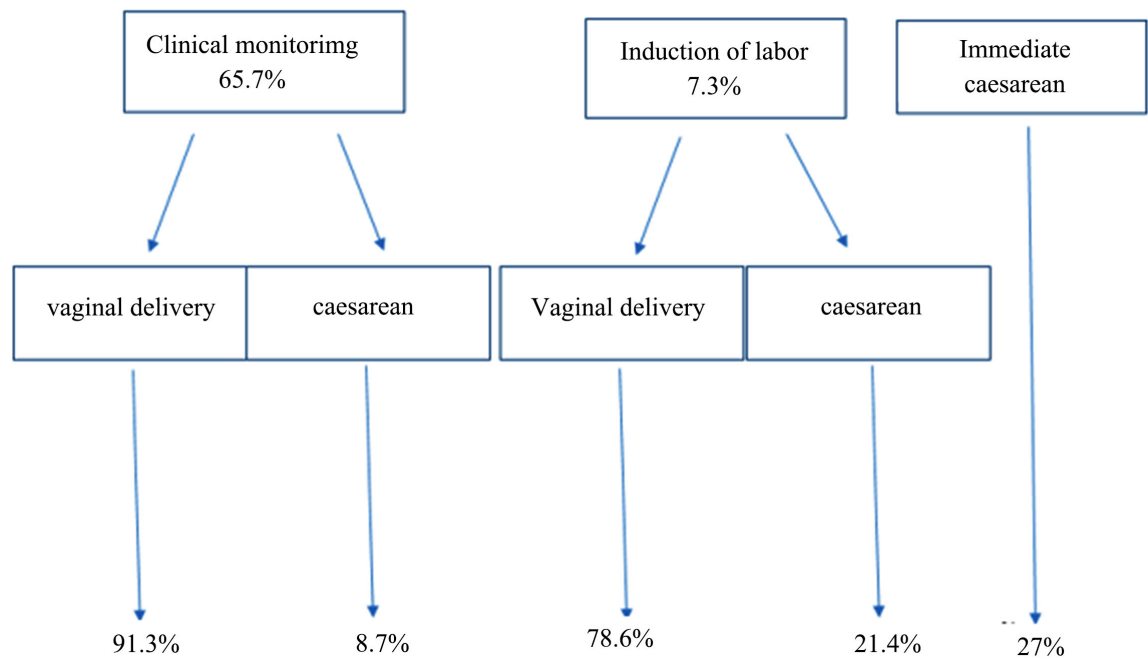

Figure 2. Type of delivery. 
Table 1. Risk factors.

\begin{tabular}{ccc}
\hline Risk factors & Actual (N) & Percentage (\%) \\
\hline Not recovered & 481 & 83.9 \\
Twins & 39 & 6.8 \\
Macrosomia & 22 & 3.8 \\
Polyhydramnios & 20 & 3.5 \\
Urogenital infections & 13 & 2.3 \\
Total & 575 & 100 \\
\hline
\end{tabular}

Table 2. Type of fetal presentation.

\begin{tabular}{ccc}
\hline Type of fotal prsentation & Numbers & Percentage \% \\
\hline Cephalic & 494 & 86 \\
Seat & 76 & 13.2 \\
Other & 5 & 0.8 \\
Total & $\mathbf{5 7 5}$ & $\mathbf{1 0 0}$ \\
\hline
\end{tabular}

\section{Complications}

In $90.4 \%$ of cases the change was normal. We found $7.5 \%$ of cases of neonatal asphyxia and $1.7 \%$ of cases of chorioamnionitis.

\section{Prognosis}

\section{Maternal prognosis}

In the postpartum period, two cases of endometritis were recorded including one case of hemorrhagic endometritis. Maternal mortality was zero.

\section{Neonatal prognosis}

In our series, 11 cases of neonatal infection were recorded either a frequency $1.8 \%$ and for these babies, antenatal antibiotic was introduced in mothers. Of these 11 infants, 6 were hospitalized in neonatal intensive care unit (NICU). Of the 610 infants, 23 (3.8\%) had died and 587 (96.2\%) were alive. Among the infants died, 16 were stillborn fresh, 2 fetal deaths in utero and 5 had died in the neonatal period.

\section{Discussion}

The PROM occurs in $5 \%$ to $10 \%$ of full-term pregnancies. The main risks are the maternal-fetal infection, prematurity and to a lesser extent the complications of oligohydramnios in case of ealy PROM. In our study $17.7 \%$ of preterm delivery were found. This frequency is close to those found in the literature. Cisse [6] and Aloulou [7] noted frequencies respectively of $25.1 \%$ and $23.19 \%$.

Another complication of PROM is chorioamnionitis which is a serious perinatal infection and this risk is increased with membrane rupture remote from term. Ten (10) of our patients had clinically and biologically confirmed 
intra-amniotic infection at a frequency of $1.8 \%$. In their study, Ramsey et al. [8] reported a clinical chorioamnionitis rate of $36 \%$ for births before 26 weeks and $6 \%$ for births to 35 - 36 SA. The incidence of neonatal infection after rupture of membranes more than 24 hours is approximately $1 \%$. When clinical signs of chorioamnionitis exist, the risk increases by $3 \%$ to $5 \%$ [9] [10]. We recorded among newborns 11 cases of neonatal infection at the frequency of $1.8 \%$. Of these neonatal infections, none were associated with cases of chorioamnionitis found. Accoceberry [2] noted $16 \%$ of cases of neonatal infection, and according to it the repetition of vaginal examinations was at the origin. Aïdibé [11] recorded only one case of neonatal infection $(0.71 \%)$ and this case was associated with chorioamnionitis.

Prescription of antibiotics in PROM before 34 reduced neonatal and maternal infectious morbidity. Indeed it drives less frequent chorioamnionitis, and reductions in neonatal morbidities including intraventricular hemorrhage, infections and the need for oxygen therapy. It is also associated with a longer latency for corticosteroids therapy [12]. However putting patients on antibiotic treatment is not systematic and is discussed by the schools. In our study $42.6 \%$ had benefited from it. The length of the rupture of membranes is a key in the antibiotic administration decision. Indeed an opening of the egg of more than 12 hours promotes contamination of amniotic fluid ascending path [9] [10]. Thus some authors justify routine antibiotic therapy for any PROM beyond 12 hours not in labor [13] [14]. Seince et al. [15] in a multicenter study found no significant difference in terms of infection, maternal-fetal according to whether the time to rupture was 6 to 12 hours, 12 to 24 hours or more. 24 hours. These same results were found in the study Mozurkeuwich and Wolf [16]. Regarding antenatal corticosteroid therapy, its importance is no longer to be proven. It leads to a reduction in neonatal deaths, respiratory distress syndrome, chronic lung disease, intraventricular haemorrhage and necrotizing enterocolitis, with no obvious increase in perinatal or maternal infections. In our study 30 patients (5.2\%) received corticosteroids.

In our series, perinatal mortality was $3.6 \%$ or 23 newborns on 610 births. Of these 23 deaths, 16 were stillbirths, 2 fetal deaths in utero while 5 had died in early neonatal period.

Among the fresh stillborn, the offending causes were severe preeclampsia (16.7\%), fetal distress (11.1\%), placental abruption (5.5\%), cord prolapse (5.5\%), and chorioamnionitis (5.5\%).

For 5 cases of neonatal deaths, there were ground for prematurity and 3 cases of neonatal infection for both.

This mortality rate is lower than those found by Aidibé [11] and Paumier [1] which recorded perinatal mortality of $1.4 \%$ and $1.45 \%$ respectively.

Thirty-four-point three percent of patients had delivery by caesarean section versus $65.7 \%$ for vaginal delivery. These results seem similar to those found themselves in literature [6] [11] [17]. 


\section{Conclusions}

These results show that the prognosis of premature rupture of membranes remains favorable in our practice.

To improve the prognosis, we recommend sensitization of patients and during prenatal consultations with regard to danger signs, of carrying out systematic bacteriological samples from all pregnant in late pregnancy and the health personnel to direct patients' references to structures in case of PROM.

\section{Conflicts of Interest}

The authors declare no conflicts of interest regarding the publication of this paper.

\section{References}

[1] Paumier, A., et al. (2008) Premature Rupture of Membranes before 32 Weeks Gestation Antenatal Prognostic Factors. Obstetrics and Gynecology, 36, 748-756.

[2] Accoceberry, M., et al. (2005) Neonatal Morbidity after Expectant Attitude Followed by a Systematic Birth to 34 Weeks of Pregnancy in Situations of Premature Rupture of Membranes. Obstetrics Gynecology \& Fertility, 33, 577-581.

[3] Pasquier, A.D. and Doret (2008) Complications and Monitoring during the Latency Period after Premature Rupture of Membranes Prior to Term: Development. Journal of Obstetrics Gynecology and Reproductive Biology, 37, 568-578.

[4] El-Messidi, A. and Cameron, A. (2010) Diagnosis of Premature Rupture of Membranes: Inspiration and Insights from the Past for the Future. Journal of Obstetrics and Gynecology Canada, 32, 561-569. https://doi.org/10.1016/S1701-2163(16)34525-X

[5] Mercer, B. (2011) Premature Rupture of the Membranes. Clinical Obstetrics and Gynecology, 54, 305-306. https://doi.org/10.1097/GRF.0b013e31821ac75d

[6] K, C. (2006) Risk Factors for Premature Rupture of Membranes in the Obstetrics and Gynecology Department of the Reference Center of the Town of Bamako District. University of Bamako, Bamako.

[7] HER (2009) Premature Rupture of Membranes: About 549 Cases. Université Cadi Ayyad, Marrakech.

[8] Ramsey, P.S., et al. (2005) Chorioamnionitis Increases Neonatal Morbidity in Pregnancies Complicated by Preterm Premature Rupture of Membranes. American Journal of Obstetrics \& Gynecology, 192, 1162-1166. https://doi.org/10.1016/j.ajog.2004.11.035

[9] Blonde, M.H., et al. (1992) Bacterial Infection of the Newborn by Maternal Fetal Contamination: One Can Depend on the Anamnesis. Obstetrics \& Gynecology (Paris), 21, 393-397.

[10] Yasmina, A. and Barakat, A. (2017) Premature Rupture of Membranes at Term: Prognostic Factors and Neonatal Consequences. The Pan African Medical Journal, 26, 68. https://doi.org/10.11604/pamj.2017.26.68.11568

[11] Aidibé, I. (2008) Management of Premature Rupture of Membranes at the Institute of Social Hygiene Dakar. In Faculty of Medicine. University Cheikh Anta Diop, Dakar.

[12] Goffinet, F. (1999) Antibiotics and Antenatal Perpartum in Case of Premature 
Rupture of Membranes. Obstetrics \& Gynecology (Paris), 28, 650-659.

[13] Cararach, V., et al. (1998) Administration of Antibiotics to Patients with Rupture of Membranes at Term, a Prospective, Randomized, Multicentric Study. Acta Obstetricia and Gynecologica Scandinavica, 77, 298-302. https://doi.org/10.1034/j.1600-0412.1998.770308.x

[14] Edwards, R.K., Locksmith, G.J. and Duff, P. (2000) Expanded-Spectrum Antibiotics with Preterm Premature Rupture of Membranes. Obstetrics \& Gynecology, 96, 60-64. https://doi.org/10.1097/00006250-200007000-00013

[15] Seince, N., et al. (2001) Management of Premature Rupture of the Membranes at Term: How Long to Delay? Results of a Multicentric Prospective Study in 713 Boxes. Obstetrics \& Gynecology (Paris), 30, 42-50.

[16] Mozurkewich, E.L. and Wolf, F.M. (1997) Premature Rupture of Membranes at Term: A Meta-Analysis of Three Management Schemes. Obstetrics \& Gynecology, 89, 1035-1043. https://doi.org/10.1016/S0029-7844(97)00094-X

[17] Andriamady, R., et al. (1999) Premature Rupture of Membranes Views to the Maternity Befelatanana, University Hospital of Antananarivo in 1998. Archives de PInstitut Pasteur de Madagascar, 65, 100-102. 